\title{
PAPER \\ Broadcast Control of Multi-Agent Systems with Quantized Measurements
}

\author{
Yosuke TANAKA $^{\dagger \mathrm{a})}$, Nonmember, Shun-ichi AZUMA ${ }^{\dagger \mathrm{b})}$, Member, and Toshiharu SUGIE ${ }^{\dagger c)}$, Nonmember $^{\circ}$
}

SUMMARY This paper addresses a broadcast control problem of multi-agent systems with quantized measurements, where each agent moves based on the common broadcasted signal and tries to minimize a given quadratic performance index. The problem is solved by introducing dither type random movements to the agents' action which reduce the degradation caused by quantized measurements. A broadcast controller is derived and it is proven that the controller approximately achieves given tasks with probability 1 . The effectiveness of the proposed controller is demonstrated by numerical simulation.

key words: multi-agent systems, broadcast control, quantization

\section{Introduction}

Broadcast control of multi-agent systems, i.e., the control of agents by sending the same signals indiscriminatingly, has become an important topic [1]-[7]. The essential feature is that the distinction of agents (ID of agents) is not required in the control scheme, and so it is promising for large-scale multi-agent systems such as swarm robot systems. Motivated by this advantage, some results have been obtained in recent years [1]-[7].

One of the standard problems, formulated in [5], is summarized as follows. Suppose that agents and a global controller are given, as shown in Fig. 1. There, the agents cannot communicate with each other, but can obtain control commands, called the broadcast signals, from the global controller via broadcast. The global controller generates the control commands by observing not the states of all the agents (vector-valued information) but scalar-valued information of the group performance. The problem of interest is to design the agents' action and the broadcast signals to achieve a given global objective.

The authors have recently presented several solutions to the problem. In [5], a basic controller and a convergence result have been presented. A more practical controller has been developed in [6], and it enables us to easily tune the controller gains for fast convergence. Moreover, the basic controller has been extended to an environment where both communication and broadcast are available [7]. On the other hand, for practical use, the broadcast control problem has to be solved with quantized measurements. For example, it is

Manuscript received July 12, 2013.

Manuscript revised October 4, 2013.

${ }^{\dagger}$ The authors are with the Graduate School of Informatics, Kyoto University, Uji-shi, 611-0011 Japan.

a)E-mail: ytanaka@ robot.kuass.kyoto-u.ac.jp

b)E-mail: sazuma@i.kyoto-u.ac.jp

c) E-mail: sugie@i.kyoto-u.ac.jp

DOI: 10.1587/transfun.E97.A.830

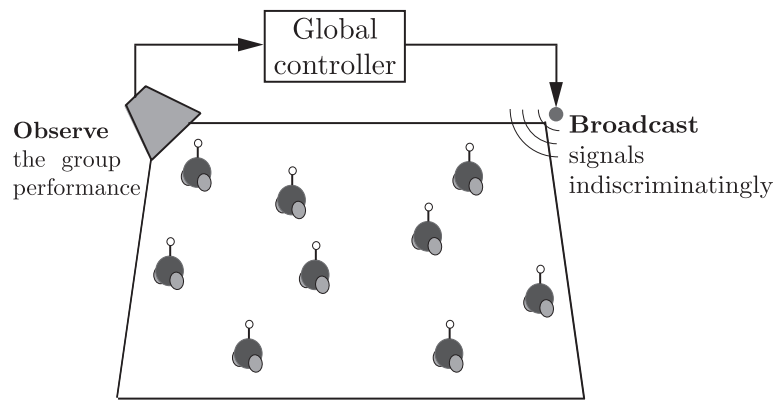

Fig. 1 Broadcast control of multi-agent systems.

typical to use a digital camera as the sensor of the global controller for estimating the performance. In such case, the measurement signal is inevitably quantized. However, no result has been obtained for such a situation.

This paper thus addresses the above broadcast control problem with quantized measurements. As the first step, we focus on the problem whose performance index is given in a quadratic form. By introducing special random movements to the agents' action for reducing the degradation caused by the quantization. Based on this idea, a broadcast controller is obtained. It is proven that the controller approximately achieves given tasks with probability 1 . The proposed controller is demonstrated by numerical simulation.

Notation: Let $\mathbf{R}, \mathbf{R}_{+}, \mathbf{Z}$, and $\mathbf{Z}_{0+}$ be the real number field, the set of positive real numbers, the set of integers, and the set of non-negative integers, respectively. We denote by $0, I_{n}$, and $\mathbf{1}_{n}$ the zero scalar/vector, the $n \times n$ identity matrix, and the $n \times 1$ vector whose all elements are one. For the vector $x$, let $\|x\|$ and $\|x\|_{\infty}$ be the Euclidean norm and the infinity norm, respectively. For the vector $x$ with nonzero elements, we use $x^{(-1)}$ to represent the elementwise inverse, e.g., $x^{(-1)}=\left[\begin{array}{ll}1 / 4 & 1 / 3\end{array}\right]^{\top}$ for $x=\left[\begin{array}{ll}4 & 3\end{array}\right]^{\top}$. The gradient of the differentiable function $J: \mathbf{R}^{n} \rightarrow \mathbf{R}$ is expressed by $\nabla J(x)$ (note $\nabla J(x) \in \mathbf{R}^{n}$ ). For the vector $x \in \mathbf{R}^{n}$, let $q(x)$ denote the elementwise uniform quantizer with the quantization interval $d \in \mathbf{R}_{+}$, as illustrated in Fig. 2. For example, $q(x)=\left[\begin{array}{lll}10 & 0 & -4\end{array}\right]$ for $x=\left[\begin{array}{lll}9.8 & 0.5 & -4.3\end{array}\right]$ and $d=1$. Finally, let $\mathbb{P}(A), \mathbb{E}(a)$, and $\mathbb{E}(a \mid b)$ respectively denote the probability of the event $A$, the expectation of the random variable $a$, and the conditional expectation of $a$ given the random variable $b$. In particular, we often use $\mathbb{E}_{a}(\cdot)$ to stress the expectation for $a$.

Finally, we prepare the following formulas:

$\left(x^{\top} y\right) z=\left(z x^{\top}\right) y$ 


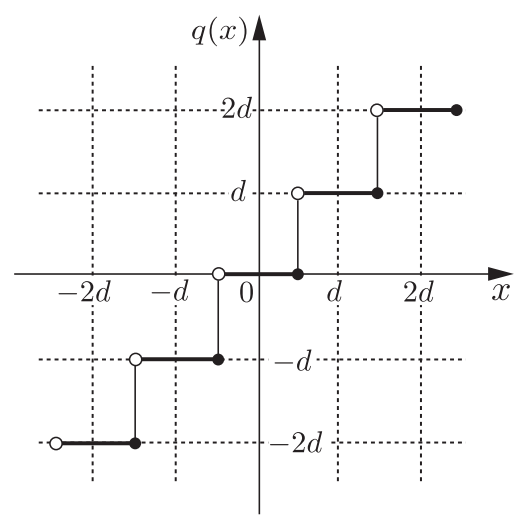

Fig. 2 Quantizer $q$ for $n=1$.

$$
\begin{aligned}
& \mathbb{E}(f(a, b) \mid c)=\mathbb{E}_{a}\left(\mathbb{E}_{b}(f(a, b) \mid a, c) \mid c\right), \\
& \mathbb{E}(g(e))=\int_{-\infty}^{\infty} g(e) p(e) d e
\end{aligned}
$$

where $x \in \mathbf{R}^{n}, y \in \mathbf{R}^{n}$, and $z \in \mathbf{R}^{n}$ are vectors, $a \in \mathbf{R}^{n}$, $b \in \mathbf{R}^{n}$, and $c \in \mathbf{R}^{n}$ are random vectors, $f: \mathbf{R}^{n} \times \mathbf{R}^{n} \rightarrow \mathbf{R}^{n}$ is a function, $e \in \mathbf{R}$ is a random number drawn from the probability distribution $p: \mathbf{R} \rightarrow\{0\} \cup \mathbf{R}_{+}$, and $g: \mathbf{R} \rightarrow \mathbf{R}$ is a function.

\section{Problem Formulation}

\subsection{System Description}

Consider the broadcast control system $\Sigma$ in Fig. 3, composed of $N$ agents, the sensor $S$, and the global controller $G$.

The physical dynamics of agent $i$ is described by

$$
P_{i}: x_{i}(t+1)=x_{i}(t)+u_{i}(t)
$$

where $t \in \mathbf{Z}_{0+}$ is the discrete time, $x_{i}(t) \in \mathbf{R}^{n}$ is the position, and $u_{i}(t) \in \mathbf{R}^{n}$ is the control input.

The local controller, which is embedded in agent $i$, is of the form

$$
L_{i}:\left\{\begin{array}{l}
\xi_{i}(t+1)=f\left(\xi_{i}(t), w(t)\right) \\
u_{i}(t)=g\left(\xi_{i}(t), w(t)\right)
\end{array}\right.
$$

where $\xi_{i}(t) \in \mathbf{R}^{\mu}$ is the state, $w(t) \in \mathbf{R}$ is the input, $u_{i}(t)$ is the output, and $f: \mathbf{R}^{\mu} \times \mathbf{R} \rightarrow \mathbf{R}^{\mu}$ and $g: \mathbf{R}^{\mu} \times \mathbf{R} \rightarrow \mathbf{R}^{n}$ are functions. The functions $f$ and $g$ and the initial state $\xi_{i}(0)$ are assumed to be the same for all the local controllers $L_{i}(i=1,2, \ldots, N)$, which means that the agents are handled indiscriminatingly.

For simplicity of notation, we denote by $x(t)$ the collective position of the agents, i.e.,

$$
x(t):=\left[\begin{array}{llll}
x_{1}^{\top}(t) & x_{2}^{\top}(t) & \cdots & x_{N}^{\top}(t)
\end{array}\right]^{\top} .
$$

In addition, the performance of the agent group is expressed by the function $J: \mathbf{R}^{n N} \rightarrow\{0\} \cup \mathbf{R}_{+}$. That is, $J$ is the performance index which quantifies the achievement degree of a task to be performed by the agents.

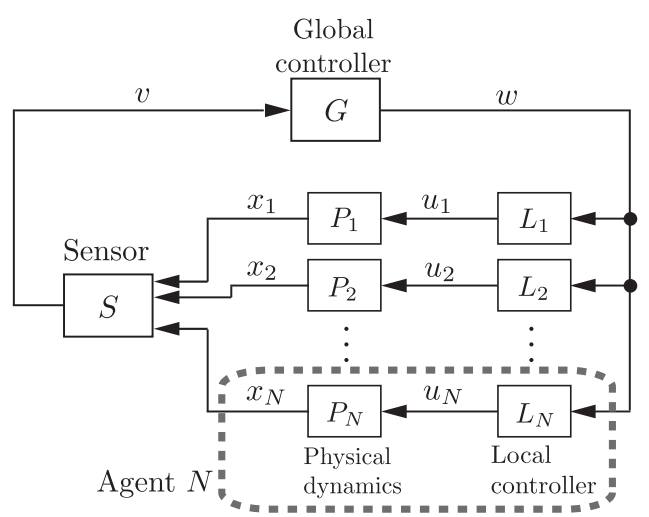

Fig. 3 Broadcast control system $\Sigma$.

The sensor $S$ corresponds to the pair of a digital camera and an image processing system, which is given by

$$
S: v(t)=J(q(x(t)))
$$

where $x(t)$ is the input and $v(t) \in\{0\} \cup \mathbf{R}_{+}$is the output. The quantizer $q$ is the model of the digital camera and $J$ is the performance index, which is interpreted as an image processing system which translates a digital image into the instantaneous value of the performance.

Finally, the global controller $G$ is given by

$$
G: w(t)=h(v(t))
$$

where $v(t)$ is the input, $w(t)$ is the output, called the broadcast signal, and $h:\{0\} \cup \mathbf{R}_{+} \rightarrow \mathbf{R}$ is a function.

2.2 Broadcast Control Problem with Quadratic Performance

In a similar way to [6], we assume here that the performance index is in the quadratic form:

$$
J(x)=x^{\top} P x+p^{\top} x+r
$$

where $P \in \mathbf{R}^{n N \times n N}$ is a positive-definite symmetric matrix, $p \in \mathbf{R}^{n N}$ is a vector, and $r \in \mathbf{R}$ is a scalar such that $J(x)>0$ for every $x \in \mathbf{R}^{n N}$. We call the above index $J(x)$ the quadratic performance index.

Then our problem is formulated as follows.

Problem 1: For the system $\Sigma$, the quantization interval $d \in$ $\mathbf{R}_{+}$and the quadratic performance index $J: \mathbf{R}^{n N} \rightarrow\{0\} \cup \mathbf{R}_{+}$ are given. Let $x^{*}$ be the solution to $\min _{x \in \mathbf{R}^{n N}} J(x)$, i.e., $x^{*}=$ $-\frac{1}{2} P^{-1} p$. Then, find local controllers $L_{i}(i=1,2, \ldots, N)$ and a global controller $G$ (i.e., find functions $f, g$, and $h$ ) such that

$$
\lim _{t \rightarrow \infty} x(t)=x^{*}
$$

for every initial states $x(0) \in \mathbf{R}^{n N}$ and $\left(\xi_{1}(0), \xi_{2}(0), \ldots\right.$, $\left.\xi_{N}(0)\right) \in \mathbf{R}^{\mu N}$ satisfying $\xi_{1}(0)=\xi_{2}(0)=\cdots=\xi_{N}(0)$.

Three remarks are given. 
First, although the class of tasks represented by (8) is somewhat limited, several major tasks are covered. For example, the formation specified by the desired positions $\hat{x}_{1}, \hat{x}_{2}, \ldots, \hat{x}_{N}$ is expressed as

$$
\begin{aligned}
J(x) & =(x-\hat{x})^{\top}(x-\hat{x}) \\
& =x^{\top} I_{n N} x-2 \hat{x}^{\top} x+\hat{x}^{\top} \hat{x}
\end{aligned}
$$

where $\hat{x}:=\left[\begin{array}{llll}\hat{x}_{1}^{\top} & \hat{x}_{2}^{\top} & \cdots & \hat{x}_{N}^{\top}\end{array}\right]^{\top} \in \mathbf{R}^{n N}$.

Second, in this problem, the task has to be achieved with limited information flow. In fact, the same signals are broadcasted to all the agents, and the measurement of the group performance (i.e., the input of $G$ ) is quantized. In particular, the quantized measurement makes the problem more challenging than the standard broadcast problem in [5].

Third, the problem seems to be related to the problem settings of the reinforcement learning, the game theory, and the simulated annealing. However, they are different from our problem. In fact, in the reinforcement learning and the game theory, the interaction among agents is assumed to be available. Moreover, the collection of optimization parameters are needed in the simulate annealing. On the other hand, it is assumed in our problem that such interaction and collection are not available, as shown in Fig. 3.

\section{Broadcast Control}

\subsection{Fundamental Properties of Quantization}

First of all, we prepare the following basic properties of the quantizer $q$ and its quantization error

$$
\delta(y):=q(y)-y
$$

where $y \in \mathbf{R}^{v}$.

(i) $q(d y)=d y$ for $y \in \mathbf{Z}^{v}$, where note that $d$ is the quantization interval defined before and $d y$ represents the product of $d$ and $y$.

(ii) $-\frac{d}{2} \mathbf{1}_{v} \leq \delta(y)<\frac{d}{2} \mathbf{1}_{v}$ for $y \in \mathbf{R}^{v}$.

(iii) $q(d y+z)=d y+q(z)$ for $y \in \mathbf{Z}^{v}$ and $z \in \mathbf{R}^{v}$.

(iv) $\delta(d y+z)=\delta(z)$ for $y \in \mathbf{Z}^{v}$ and $z \in \mathbf{R}^{v}$.

The former three properties are trivial by the definitions of $q$ and $\delta$. The final property is straightforwardly proven by (10), (i), and (iii), i.e., $\delta(d y+z)=q(d y+z)-(d y+z)=$ $d y+q(z)-(d y+z)=q(z)-z=\delta(z)$.

\subsection{Proposed Controller}

In this paper, we propose $L_{i}(i=1,2, \ldots, N)$ and $G$ given by (5), (7), and

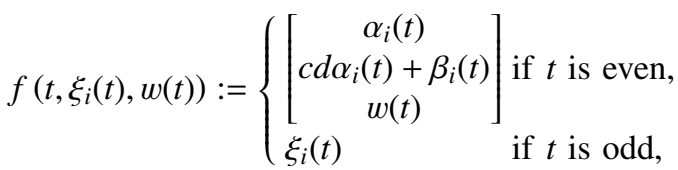

$g\left(t, \xi_{i}(t), w(t)\right)$

$$
:= \begin{cases}c d \alpha_{i}(t)+\beta_{i}(t) & \text { if } t \text { is even, } \\ -\xi_{i 2}(t)-a(t) \frac{w(t)-\xi_{i 3}(t)}{c d} \xi_{i 1}^{(-1)}(t) & \text { if } t \text { is odd, }\end{cases}
$$

$h(v(t)):=v(t)$.

Here, $\xi_{i 1}(t) \in \mathbf{R}^{n}, \xi_{i 2}(t) \in \mathbf{R}^{n}$, and $\xi_{i 3}(t) \in \mathbf{R}$ are the components of the state $\xi_{i}(t) \in \mathbf{R}^{2 n+1}$, i.e.,

$$
\xi_{i}(t)=\left[\begin{array}{l}
\xi_{i 1}(t) \\
\xi_{i 2}(t) \\
\xi_{i 3}(t)
\end{array}\right]
$$

$\xi_{i 1}^{(-1)}(t) \in \mathbf{R}^{n}$ is the elementwise inverse of $\xi_{i 1}(t)$ as defined at the end of Sect. $1, \alpha_{i}(t) \in \mathbf{R}^{n}$ and $\beta_{i}(t) \in \mathbf{R}^{n}$ are random vectors, and $a(t) \in \mathbf{R}_{+}$and $c \in \mathbf{R}_{+}$(c is constant) are the gains.

The proposed controller set works as follows. The global controller $G$ sends the achievement degree of the task at every $t$. The local controllers $L_{i}(i=1,2, \ldots, N)$, on the other hand, let each agent alternately perform the following two types of movement, as illustrated in Fig. 4. One is the random movement to $x_{i}(t)+c d \alpha_{i}(t)+\beta_{i}(t)$ at even $t$. The other is the deterministic movement based on the difference of the achievement degree at odd $t$.

Although the proposed controllers are similar to those of [5]-[7], the local controllers are slightly different in the sense that the random vectors $\beta_{i}(t)(i=1,2, \ldots, N)$ are introduced. The random vectors $\beta_{i}(t)$ play a role of reducing the degradation caused by the quantization, which is a key idea of this paper. This fact is clarified in the following lemma.

Lemma 1: For the system $\Sigma$, suppose that the quantization interval $d$ and the quadratic performance index $J$ are given. Suppose also that $L_{i}(i=1,2, \ldots, N)$ and $G$ are given by (5), (7), and (11)-(13). Then

$$
\mathbb{E}(\delta(x(2 s+1)) \mid x(2 s))=0
$$

holds for every $s \in \mathbf{Z}_{0+}$ subject to the following conditions:

(A1) $\alpha_{i j}(t)(i=1,2, \ldots, N, j=1,2, \ldots, n, t=0,1, \ldots)$ are i.i.d. random numbers drawn from the Bernoulli distribution

$$
\left\{\begin{array}{l}
\mathbb{P}\left(\alpha_{i j}(t)=1\right)=0.5, \\
\mathbb{P}\left(\alpha_{i j}(t)=-1\right)=0.5
\end{array}\right.
$$

where $\alpha_{i j}(t)$ is the $j$-th element of $\alpha_{i}(t)$.

(A2) $\beta_{i j}(t)(i=1,2, \ldots, N, j=1,2, \ldots, n, t=0,1, \ldots)$ are i.i.d. random numbers drawn from the uniform distribution on $(-d / 2, d / 2]$ in Fig. 5, where $\beta_{i j}(t)$ is the $j$-th element of $\beta_{i}(t)$ and $d$ is the quantization interval of the quantizer $q$, as defined before.

(A3) $c$ is a positive integer.

Proof: Let $x_{i j}(t)(j=1,2, \ldots, n)$ be the $j$-th element of $x_{i}(t)$. Then the following relation between $x_{i j}(2 s)$ and $x_{i j}(2 s+1)\left(s \in \mathbf{Z}_{0+}\right)$ is obtained from (4), (5), (7), and (11)(13): 

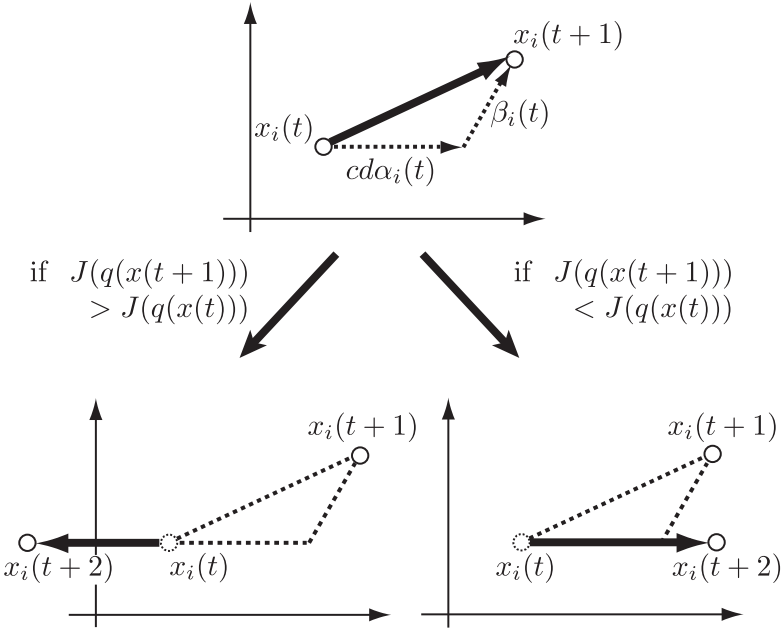

Fig. 4 Agent motion by $L_{i}$ for even $t$.

$$
x_{i j}(2 s+1)=x_{i j}(2 s)+c d \alpha_{i j}(2 s)+\beta_{i j}(2 s) \text {. }
$$

From (10) and (15), $\delta\left(x_{i j}(2 s+1)\right)$ is expressed as

$$
\begin{aligned}
\delta & \left(x_{i j}(2 s+1)\right) \\
& =\delta\left(x_{i j}(2 s)+c d \alpha_{i j}(2 s)+\beta_{i j}(2 s)\right) \\
& =\delta\left(q\left(x_{i j}(2 s)\right)-\delta\left(x_{i j}(2 s)\right)+c d \alpha_{i j}(2 s)+\beta_{i j}(2 s)\right) \\
& =\delta\left(d\left(\frac{q\left(x_{i j}(2 s)\right)}{d}+c \alpha_{i j}(2 s)\right)-\delta\left(x_{i j}(2 s)\right)+\beta_{i j}(2 s)\right) \\
& =\delta\left(-\delta\left(x_{i j}(2 s)\right)+\beta_{i j}(2 s)\right) .
\end{aligned}
$$

The last equality follows from the property (iv) of $q$ and the relation $q\left(x_{i j}(2 s)\right) / d+c \alpha_{i j}(2 s) \in \mathbf{Z}$ which is obtained from (A1), (A3), and the definition of $q$.

Since the property (ii) of $q$ implies $-d / 2<-\delta\left(x_{i j}(2 s)\right)$ $\leq d / 2$, and the probability distribution in Fig. 5 , specified by the quantization interval $d$, is used, we have

$$
\begin{aligned}
& \delta\left(x_{i j}(2 s+1)\right)= \\
& \begin{cases}\delta\left(x_{i j}(2 s)\right)-\beta_{i j}(2 s) & \text { if }-\frac{d}{2} \leq \beta_{i j}(2 s) \leq \frac{d}{2}+\delta\left(x_{i j}(2 s)\right), \\
d+\delta\left(x_{i j}(2 s)\right)-\beta_{i j}(2 s) & \text { if } \frac{d}{2}+\delta\left(x_{i j}(2 s)\right)<\beta_{i j}(2 s) \leq \frac{d}{2},\end{cases} \\
& \text { if } 0 \leq-\delta\left(x_{i j}(2 s)\right) \leq \frac{d}{2}, \\
& \delta\left(x_{i j}(2 s+1)\right)=\quad \text { if }-\frac{d}{2}+\delta\left(x_{i j}(2 s)\right)<\beta_{i j}(2 s) \leq \frac{d}{2}, \\
& -d+\delta\left(x_{i j}(2 s)\right)-\beta_{i j}(2 s) \text { if }-\frac{d}{2} \leq \beta_{i j}(2 s) \leq-\frac{d}{2}+\delta\left(x_{i j}(2 s)\right),
\end{aligned}
$$

whose proofs are shown in Appendix A.

By noting that $\delta\left(x_{i j}(2 s+1)\right)$ is a function of $\beta_{i j}(2 s)$, it follows from (A1), (A2), and (16)-(18) that

$\mathbb{E}\left(\delta\left(x_{i j}(2 s+1)\right) \mid x(2 s)\right)$

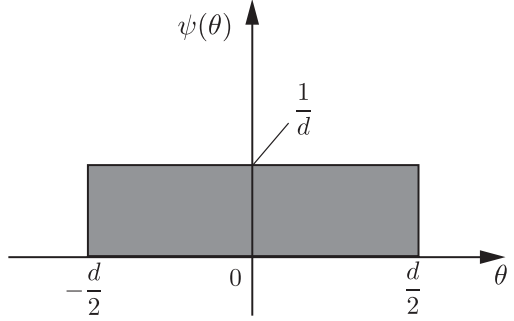

Fig. 5 Probability distribution of $\beta_{i j}(t)$.

$$
\begin{aligned}
& =\mathbb{E}_{\alpha_{i j}}\left(\mathbb{E}_{\beta_{i j}}\left(\delta\left(x_{i j}(2 s+1)\right) \mid x(2 s), \alpha_{i j}(2 s)\right) \mid x(2 s)\right) \\
& =\mathbb{E}_{\alpha_{i j}}\left(\left.\int_{-d / 2}^{d / 2} \delta\left(x_{i j}(2 s+1)\right)\right|_{\beta_{i j}(2 s)=\theta} \psi(\theta) d \theta \mid x(2 s)\right)
\end{aligned}
$$

$$
=\left\{\begin{array}{c}
\mathbb{E}_{\alpha_{i j}}\left(\int_{-d / 2}^{d / 2+\delta\left(x_{i j}(2 s)\right)}\left(\delta\left(x_{i j}(2 s)\right)-\theta\right) \frac{1}{d} d \theta\right. \\
\left.+\int_{d / 2+\delta\left(x_{i j}(2 s)\right)}^{d / 2}\left(d+\delta\left(x_{i j}(2 s)\right)-\theta\right) \frac{1}{d} d \theta \mid x(2 s)\right) \\
\text { if } 0 \leq-\delta\left(x_{i j}(2 s)\right) \leq \frac{d}{2}, \\
\mathbb{E}_{\alpha_{i j}}\left(\int_{-d / 2}^{-d / 2+\delta\left(x_{i j}(2 s)\right)}\left(-d+\delta\left(x_{i j}(2 s)\right)-\theta\right) \frac{1}{d} d \theta\right. \\
\left.+\int_{-d / 2+\delta\left(x_{i j}(2 s)\right)}^{d / 2}\left(\delta\left(x_{i j}(2 s)\right)-\theta\right) \frac{1}{d} d \theta \mid x(2 s)\right) \\
\text { if }-\frac{d}{2}<-\delta\left(x_{i j}(2 s)\right)<0
\end{array}\right.
$$

$=\mathbb{E}_{\alpha_{i j}}(0 \mid x(2 s))$

$=0$,

where (2) and (3) are utilized. By putting (19) for $i=$ $1,2, \ldots, N$ and $j=1,2, \ldots, n$ together, we have (14).

Lemma 1 shows that the expectation of the quantization error for $x(2 s+1)$ is zero. This fact presents the following result.

Lemma 2: For the system $\Sigma$, suppose that the quantization interval $d$ and the quadratic performance index $J$ are given. Suppose also that $L_{i}(i=1,2, \ldots, N)$ and $G$ are given by (5), (7), and (11)-(13). If (A1)-(A3) hold, then

$\mathbb{E}\left(\frac{w(2 s+1)-w(2 s)}{c d} \alpha^{(-1)}(2 s) \mid x(2 s)\right)=\frac{\partial J(x(2 s))}{\partial x}$

for every $s \in \mathbf{Z}_{0+}$.

Proof: Let $\alpha(t):=\left[\begin{array}{llll}\alpha_{1}^{\top}(t) & \alpha_{2}^{\top}(t) & \cdots & \alpha_{N}^{\top}(t)\end{array}\right]^{\top} \in \mathbf{R}^{n N}$ and $\beta(t):=\left[\beta_{1}^{\top}(t) \beta_{2}^{\top}(t) \cdots \beta_{N}^{\top}(t)\right]^{\top} \in \mathbf{R}^{n N}$. These enable us to represent (15) as

$$
x(2 s+1)=x(2 s)+c d \alpha(2 s)+\beta(2 s) .
$$

Moreover, for simplicity of notation, we often use $x, \delta, \delta_{+}$, $\alpha$, and $\beta$ to express $x(2 s), \delta(x(2 s)), \delta(x(2 s+1)), \alpha(2 s)$, and $\beta(2 s)$.

Next, from (6)-(8), (13), and (21), we have the following equations for $w(2 s)$ and $w(2 s+1)$ : 


$$
\begin{aligned}
& w(2 s)=J(q(x(2 s))) \\
& =J(x+\delta) \\
& =(x+\delta)^{\top} P(x+\delta)+p^{\top}(x+\delta)+r \\
& \begin{aligned}
w(2 s+1)= & J(q(x(2 s+1))) \\
= & J\left(x+c d \alpha+\beta+\delta_{+}\right) \\
= & \left(x+c d \alpha+\beta+\delta_{+}\right)^{\top} P\left(x+c d \alpha+\beta+\delta_{+}\right) \\
& +p^{\top}\left(x+c d \alpha+\beta+\delta_{+}\right)+r .
\end{aligned}
\end{aligned}
$$

From (22) and (23), the left-hand side of (20) is represented as

$$
\begin{aligned}
& \mathbb{E}\left(\frac{w(2 s+1)-w(2 s)}{c d} \alpha^{(-1)}(2 s) \mid x(2 s)\right) \\
& =\mathbb{E}\left(\left(A+\alpha^{\top}(2 s) B+C \alpha(2 s)+\alpha^{\top}(2 s) D \alpha(2 s)\right) \alpha^{(-1)}(2 s) \mid x(2 s)\right)
\end{aligned}
$$

for

$$
\begin{aligned}
A:= & \frac{1}{c d}\left(\left(x+\beta+\delta_{+}\right)^{\top} P\left(x+\beta+\delta_{+}\right)\right. \\
& \left.-(x+\delta)^{\top} P(x+\delta)+p^{\top}\left(\beta+\delta_{+}-\delta\right)\right), \\
B:= & P\left(x+\beta+\delta_{+}\right), \\
C:= & \left(x+\beta+\delta_{+}\right)^{\top} P+p^{\top}, \\
D:= & c d P .
\end{aligned}
$$

Then Lemma 1, (A1), (A2), (25)-(28), and the fact that $\delta(x(2 s+1))$ is independent from $\alpha^{(-1)}(2 s)$ (which follows from (16) and (A2)) give the following equations:

$$
\begin{aligned}
& \mathbb{E}\left(A \alpha^{(-1)}(2 s) \mid x(2 s)\right)=0, \\
& \mathbb{E}\left(\left(\alpha^{\top}(2 s) B\right) \alpha^{(-1)}(2 s) \mid x(2 s)\right)=P x, \\
& \mathbb{E}\left(C \alpha(2 s) \alpha^{(-1)}(2 s) \mid x(2 s)\right)=P x+p, \\
& \mathbb{E}\left(\left(\alpha^{\top}(2 s) D \alpha(2 s)\right) \alpha^{(-1)}(2 s) \mid x(2 s)\right)=0 .
\end{aligned}
$$

Their proofs are given in Appendix B.

From (8), (24), and (29)-(32), the left-hand side of (20) becomes

$$
\begin{aligned}
\mathbb{E}\left(\frac{w(2 s+1)-w(2 s)}{c d} \alpha^{(-1)}(2 s) \mid x(2 s)\right) & =2 P x(2 s)+p \\
& =\frac{\partial J(x(2 s))}{\partial x} .
\end{aligned}
$$

In this way, (20) is proven.

Lemma 2 provides a convergence result for the proposed controller given by (5), (7), and (11)-(13).

Theorem 1: For the system $\Sigma$, suppose that the quantization interval $d$ and the quadratic performance index $J$ are given. Suppose also that $L_{i}(i=1,2, \ldots, N)$ and $G$ are given by (5), (7), and (11)-(13). Then

$$
\limsup _{t \rightarrow \infty}\left\|x(t)-x^{*}\right\| \leq\left(c+\frac{1}{2}\right) d \sqrt{n N} \quad \text { w.p. } 1
$$

if (A1)-(A3) and the following conditions hold:

$$
\begin{aligned}
& \text { (B1) } \lim _{t \rightarrow \infty} a(t)=0, \sum_{t=0}^{\infty} a(t)=\infty \text {, and } \sum_{t=0}^{\infty} a^{2}(t)<\infty . \\
& \text { (B2) } \sup _{t \in \mathbf{Z}_{0+}}\|x(t)\|<\infty \text { w.p.1. }
\end{aligned}
$$

Proof: Equations (4), (5), (11), and (12) provide

$$
x(2 s+2)=x(2 s)-a(2 s+1) \phi(2 s+1)
$$

where

$$
\phi(2 s+1):=\frac{w(2 s+1)-w(2 s)}{c d} \alpha^{(-1)}(2 s) .
$$

Next, let us introduce the new variable

$$
e(2 s+1):=\phi(2 s+1)-\mathbb{E}(\phi(2 s+1) \mid x(2 s)) .
$$

Applying (35), (36), and Lemma 2 to (34), we have

$x(2 s+2)=x(2 s)-a(2 s+1) \nabla J(x(2 s))-a(2 s+1) e(2 s+1)$.

This corresponds to a stochastic approximation algorithm, called the Robbins-Monro algorithm, which is shown in Appendix $\mathrm{C}$. In addition, it can be proven that the conditions for the convergence, i.e., (C1)-(C4) in the appendix, hold subject to (A1), (B1), and (B2), as shown in Appendix D. So, we have

$$
\lim _{s \rightarrow \infty}\left\|x(2 s)-x^{*}\right\|=0 \quad \text { w.p. } 1 \text {. }
$$
that

On the other hand, from (A1), (A2), and (21), it follows

$$
\begin{aligned}
\|x(2 s+1)-x(2 s)\| & =\|c d \alpha(2 s)+\beta(2 s)\| \\
& \leq c d\|\alpha(2 s)\|+\|\beta(2 s)\| \\
& \leq\left(c+\frac{1}{2}\right) d \sqrt{n N} .
\end{aligned}
$$

Equations (38) and (39) imply (33).

Theorem 1 presents an approximate solution to Problem 1 in the sense of (33). Equation (33) shows the convergence not to $x^{*}$, i.e., the minimum of $J(x)$, but to a region including $x^{*}$. This is a reasonable solution to our problem, because the measurements are quantized as in (6) and the convergence to a single point is not possible.

Since the right-hand side of (33) depends on the number $N$ of agents, it seems that the performance will be worse as $N$ increases. However, it is not true. In fact, it follows from (A1), (A2), (15), and (38) that (33) implies

$$
\limsup _{t \rightarrow \infty}\left\|x_{i}(t)-x_{i}^{*}\right\| \leq\left(c+\frac{1}{2} d\right) \sqrt{n}
$$

where $x_{i}^{*} \in \mathbf{R}^{n}(i=1,2, \ldots, N)$ is the vector such that $x^{*}=\left[\begin{array}{llll}x_{1}^{* \top} & x_{2}^{* \top} \cdots & x_{N}^{* \top}\end{array}\right]^{\top}$. That is, from the viewpoint of each agent's position, the performance does not depend on the number $N$. 


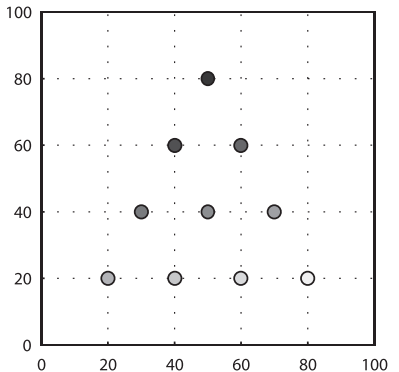

(a) Desired formation specified by $\hat{x}_{1}, \hat{x}_{2}, \ldots, \hat{x}_{10}$

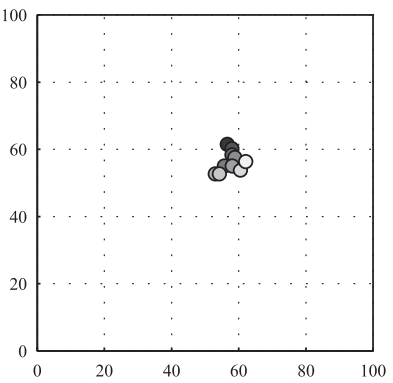

(b) Initial formation.

Fig. 6 Desired and initial formations.

Remark 1: Note in (12) that the term

$$
\frac{w(t)-\xi_{i 3}(t)}{c d} \xi_{i 1}^{(-1)}(t)
$$

corresponds to an approximation of the gradient of $J(x)$. In fact, since (4)-(7) and (11)-(13) imply $w(t)=J(q(x(t-1)+$ $c d \alpha(t-1)+\beta(t-1))), \xi_{i 3}(t)=J(q(x(t-1)))$, and $\xi_{i 1}^{(-1)}(t)=$ $\alpha_{i}^{(-1)}(t)$, we have

$$
\frac{w(t)-\xi_{i 3}(t)}{c d} \xi_{i 1}^{(-1)}(t)=\left[\begin{array}{c}
\frac{J(q(x(t-1)+c d \alpha(t-1)+\beta(t-1)))-J(q(x(t-1)))}{c d \alpha_{i 1}(t-1)} \\
\frac{J(q(x(t-1)+c d \alpha(t-1)+\beta(t-1)))-J(q(x(t-1)))}{c d \alpha_{i 2}(t-1)} \\
\vdots \\
\frac{J(q(x(t-1)+c d \alpha(t-1)+\beta(t-1)))-J(q(x(t-1)))}{c d \alpha_{i n}(t-1)}
\end{array}\right] .
$$

Therefore, by regarding $\alpha$ (i.e., $\alpha_{i j}$ ) as a perturbation parameter, the proposed controller can be interpreted as a gradientbased controller.

\section{Example}

Consider the system $\Sigma$ with $N:=10, n:=2$, and $d:=3$. We address here the formation problem. The performance in$\operatorname{dex} J(x)$ is of (9), where the desired positions $\hat{x}_{1}, \hat{x}_{2}, \ldots, \hat{x}_{10}$ and the initial positions $x_{1}(0), x_{2}(0), \ldots, x_{10}(0)$ are given as Fig. 6.

We use the controllers $L_{i}(i=1,2, \ldots, 10)$ and $G$ given by (5), (7), and (11)-(13). The gains of $L_{i}$ are set as

$$
a(t):=\frac{0.95}{(t+30)^{0.99}}, \quad c:=1,
$$

which satisfy (A3) and (B1). Moreover, the probability distributions of $\alpha_{i}(t)$ and $\beta_{i}(t)$ are given so as to satisfy (A1) and (A2). (B2) is approximately verified by the Monte Carlo method. Then we have the following result from Theorem 1:

$$
\limsup _{t \rightarrow \infty}\|x(t)-\hat{x}\| \leq 20.1 \quad \text { w.p. } 1,
$$

where $x^{*}=\hat{x}$.

Figure 7 illustrates the snapshots of the agent positions
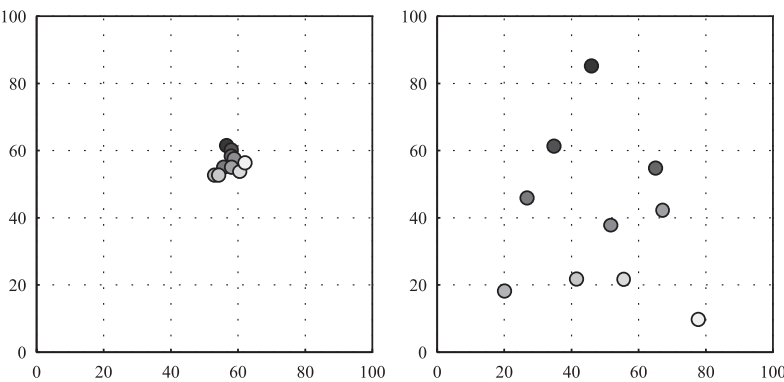

(a) $t=0$

(b) $t=200$.
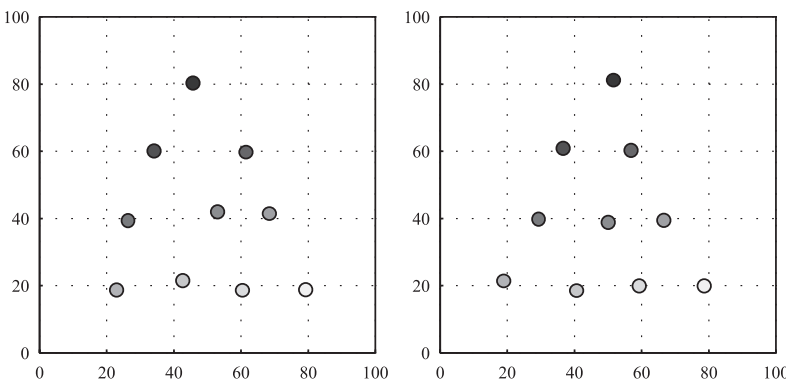

(c) $t=400$.

(d) $t=600$
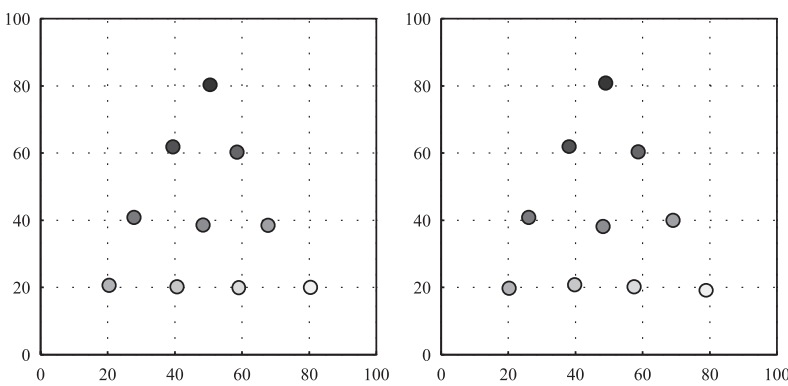

(e) $t=800$.

(f) $t=1000$.

Fig. 7 Snapshots of agent positions.

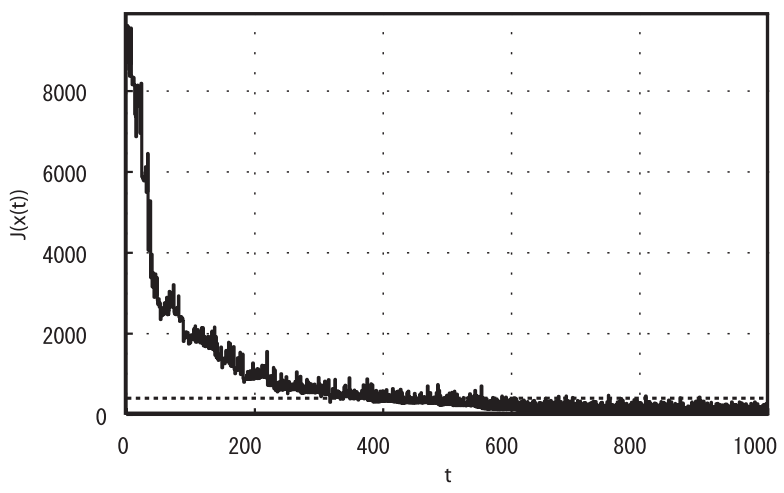

Fig. 8 Time evolution of the performance index.

$x_{i}(t)(i=1,2, \ldots, N)$ at $t=0,200, \ldots, 1000$. On the other hand, the time evolution of the performance index $J(x(t))$ is depicted by the solid line in Fig. 8, while the dotted line shows the value of the right-hand side of (42). These show that the proposed controllers achieve the desired formation under the quantized measurements. 


\section{Conclusion}

This paper presents a broadcast control method with quantized measurements. By introducing special random movements, we have obtained a broadcast controller to achieve given tasks whose performance index is given in a quadratic form. In addition, as a theoretical result, the almost sure convergence for the proposed controller has been also proven. Finally, it has been shown by numerical simulation that the task is successfully achieved by the proposed controller.

\section{Acknowledgments}

This work was partly supported by Grant-in-Aid for Scientific Research (A) 25249058 and Grant-in-Aid for Young Scientists (A) 24686051 from the Ministry of Education, Culture, Sports, Science and Technology of Japan.

\section{References}

[1] J. Ueda, L. Odhner, and H. Asada, "Broadcast feedback of stochastic cellular actuators inspired by biological muscle control," Int. J. Robotics Research, vol.26, no.11-12, pp.1251-1265, 2007.

[2] A.A. Julius, A. Halasz, M.S. Saker, H. Rubin, V. Kumar, and G.J. Pappas, "Stochastic modeling and control of biological systems: The lactose regulation system of Escherichia coli," IEEE Trans. Autom. Control, vol.53, no.1, pp.51-65, 2008.

[3] K. Das and D. Ghose, "Positional consensus in multi-agent systems using a broadcast control mechanism," 2009 American Control Conference, pp.5731-5736, 2009.

[4] S. Berman, A. Halasz, M. Ani Hsieh, and V. Kumar, "Optimized stochastic policies for task allocation in swarms of robots," IEEE Trans. Robotics, vol.25, no.4, pp.927-937, 2009.

[5] S. Azuma, R. Yoshimura, and T. Sugie, "Broadcast control of multiagent systems," Automatica, vol.49, no.8, pp.2307-2316, 2013.

[6] Y. Tanaka, S. Azuma, and T. Sugie, "Broadcast control with constantdistance random movement," Trans. Society of Instrument and Control Engineers (in Japanese), vol.50, no.2, 2014, to appear.

[7] S. Azuma, Y. Tanaka, and T. Sugie, "Multi-agent consensus under communication-broadcast mixed environment," 51st IEEE Conference on Decision and Control, pp.94-99, 2012.

[8] H.J. Kushner and D.S. Clark, "Stochastic approximation methods for constrained and unconstrained systems," Springer, 1978.

\section{Appendix A: Proofs of (17) and (18)}

Noting $-d / 2<-\delta\left(x_{i j}(2 s)\right) \leq d / 2$ from (ii) of the property of $q$, we show $\delta\left(x_{i j}(2 s+1)\right)$ by dividing into two cases:

$$
0 \leq-\delta\left(x_{i j}(2 s)\right) \leq \frac{d}{2}
$$

and

$$
-\frac{d}{2}<-\delta\left(x_{i j}(2 s)\right)<0 .
$$

First, consider the former case. Condition (A2) and (A. 1) imply $-d / 2<-\delta\left(x_{i j}(2 s)\right)+\beta_{i j}(2 s) \leq d$. Then it turns out that $q\left(-\delta\left(x_{i j}(2 s)\right)+\beta_{i j}(2 s)\right) \in\{0, d\}$ by the definition of $q$. From this fact, (10), and (16), we obtain

$$
\begin{aligned}
& \delta\left(x_{i j}(2 s+1)\right) \\
& =\delta\left(-\delta\left(x_{i j}(2 s)\right)+\beta_{i j}(2 s)\right) \\
& =q\left(-\delta\left(x_{i j}(2 s)\right)+\beta_{i j}(2 s)\right)+\delta\left(x_{i j}(2 s)\right)-\beta_{i j}(2 s) \\
& = \begin{cases}\delta\left(x_{i j}(2 s)\right)-\beta_{i j}(2 s) & \text { if } q\left(-\delta\left(x_{i j}(2 s)\right)+\beta_{i j}(2 s)\right)=0, \\
d+\delta\left(x_{i j}(2 s)\right)-\beta_{i j}(2 s) & \text { if } q\left(-\delta\left(x_{i j}(2 s)\right)+\beta_{i j}(2 s)\right)=d .\end{cases}
\end{aligned}
$$

Moreover, it follows from (A2) and (A. 1) that

$$
\begin{aligned}
& q\left(-\delta\left(x_{i j}(2 s)\right)+\beta_{i j}(2 s)\right)=0 \\
& \Leftrightarrow-\frac{d}{2}<-\delta\left(x_{i j}(2 s)\right)+\beta_{i j}(2 s) \leq \frac{d}{2} \\
& \Leftrightarrow-\frac{d}{2}+\delta\left(x_{i j}(2 s)\right)<\beta_{i j}(2 s) \leq \frac{d}{2}+\delta\left(x_{i j}(2 s)\right) \\
& \Leftrightarrow-\frac{d}{2}<\beta_{i j}(2 s) \leq \frac{d}{2}+\delta\left(x_{i j}(2 s)\right), \\
& q\left(-\delta\left(x_{i j}(2 s)\right)+\beta_{i j}(2 s)\right)=d \\
& \Leftrightarrow \frac{d}{2}<-\delta\left(x_{i j}(2 s)\right)+\beta_{i j}(2 s) \leq \frac{3 d}{2} \\
& \Leftrightarrow \frac{d}{2}+\delta\left(x_{i j}(2 s)\right)<\beta_{i j}(2 s) \leq \frac{3 d}{2}+\delta\left(x_{i j}(2 s)\right) \\
& \Leftrightarrow \frac{d}{2}+\delta\left(x_{i j}(2 s)\right)<\beta_{i j}(2 s) \leq \frac{d}{2} .
\end{aligned}
$$

Note here that the final equivalence relations of (A.4) and (A.5) are given by the fact that $\beta_{i j}(2 s) \in(-d / 2, d / 2]$ in (A2). Equations (A.3)-(A.5) imply (17).

Likewise, (18) can be proven by considering the case $(\mathrm{A} \cdot 2)$.

Appendix B: Proofs of (29)-(32)

\section{B.1 Proof of (29)}

From (A1) (which implies $\alpha_{i j}(t)=\alpha_{i j}^{-1}(t)$ ), we obtain

$$
\begin{aligned}
\mathbb{E}\left(\alpha^{(-1)}(2 s) \mid x(2 s)\right) & =\mathbb{E}(\alpha(2 s) \mid x(2 s)) \\
& =0 .
\end{aligned}
$$

Moreover, since (A1), (A2), (16), and (25), $A$ is independent from $\alpha(2 s)$. These facts and (2) imply

$$
\begin{aligned}
\mathbb{E}\left(A \alpha^{(-1)}(2 s) \mid x(2 s)\right) & =\mathbb{E}_{A}\left(\mathbb{E}_{\alpha}\left(A \alpha^{(-1)}(2 s) \mid x(2 s), A\right) \mid x(2 s)\right) \\
& =\mathbb{E}_{A}\left(A \mathbb{E}_{\alpha}\left(\alpha^{(-1)}(2 s) \mid x(2 s), A\right) \mid x(2 s)\right) \\
& =\mathbb{E}_{A}(0 \mid x(2 s)) \\
& =0,
\end{aligned}
$$

which proves (29).

B.2 Proofs of (30) and (31)

Since (A1) implies 


$$
\mathbb{E}\left(\alpha^{(-1)}(2 s) \alpha^{\top}(2 s) \mid x(2 s)\right)=I_{n N}
$$

and $B$ is independent from $\alpha(2 s)$, it turns out from (A2), Lemma 1, (1), (2), and (26) that

$$
\begin{aligned}
& \mathbb{E}\left(\left(\alpha^{\top}(2 s) B\right) \alpha^{(-1)}(2 s) \mid x(2 s)\right) \\
& =\mathbb{E}\left(\left(\alpha^{(-1)}(2 s) \alpha^{\top}(2 s)\right) B \mid x(2 s)\right) \\
& =\mathbb{E}_{B}\left(\mathbb{E}_{\alpha}\left(\left(\alpha^{(-1)}(2 s) \alpha^{\top}(2 s)\right) B \mid x(2 s), B\right) \mid x(2 s)\right) \\
& =\mathbb{E}_{B}\left(\mathbb{E}_{\alpha}\left(\alpha^{(-1)}(2 s) \alpha^{\top}(2 s) \mid x(2 s), B\right) B \mid x(2 s)\right) \\
& =\mathbb{E}_{B}(B \mid x(2 s)) \\
& =\mathbb{E}(P(x(2 s)+\beta(2 s)+\delta(x(2 s+1))) \mid x(2 s)) \\
& =P x(2 s),
\end{aligned}
$$

which proves (30).

In a similar way, (31) is proven.

\section{B.3 Proof of (32)}

Let $\hat{\alpha}_{i}$ be the $i$-th element of $\alpha$, i.e., $\alpha_{i j}=\hat{\alpha}_{(i-1) n+j}$. Then, (A1) gives

$$
\begin{aligned}
& \mathbb{E}\left(\hat{\alpha}_{i}(2 s) \hat{\alpha}_{j}(2 s) \hat{\alpha}_{k}^{-1}(2 s)\right) \\
& = \begin{cases}\mathbb{E}\left(\hat{\alpha}_{k}^{-1}(2 s)\right) & \text { if } i=j, \\
\mathbb{E}\left(\hat{\alpha}_{i}(2 s)\right) & \text { if } i \neq j=k, \\
\mathbb{E}\left(\hat{\alpha}_{j}(2 s)\right) & \text { if } j \neq i=k, \\
\mathbb{E}\left(\hat{\alpha}_{i}(2 s)\right) \mathbb{E}\left(\hat{\alpha}_{j}(2 s)\right) \mathbb{E}\left(\hat{\alpha}_{k}^{-1}(2 s)\right) & \text { otherwise }\end{cases}
\end{aligned}
$$

for every $(i, j, k) \in\{1,2, \ldots, n N\}^{3}$, and the right-hand side of (A. 7) is equal to 0 from (A.6). And also, $D$ is independent from $\alpha(2 s)$. By using these facts, the $k$-th element of (32) is obtained as follows:

$$
\begin{aligned}
& \mathbb{E}\left(\left(\alpha^{\top}(2 s) D \alpha(2 s)\right) \hat{\alpha}_{k}^{-1}(2 s) \mid x(2 s)\right) \\
& =\mathbb{E}_{D}\left(\sum_{i=1}^{n N} \sum_{j=1}^{n N} \mathbb{E}_{\alpha}\left(\hat{\alpha}_{i}(2 s) D_{i j} \hat{\alpha}_{j}(2 s) \hat{\alpha}_{k}^{-1}(2 s) \mid x(2 s), D\right) \mid x(2 s)\right) \\
& =\mathbb{E}_{D}\left(\sum_{i=1}^{n N} \sum_{j=1}^{n N} D_{i j} \mathbb{E}_{\alpha}\left(\hat{\alpha}_{i}(2 s) \hat{\alpha}_{j}(2 s) \hat{\alpha}_{k}^{-1}(2 s) \mid x(2 s), D\right) \mid x(2 s)\right) \\
& =\mathbb{E}_{D}\left(\sum_{i=1}^{n N} \sum_{j=1}^{n N} 0 \mid x(2 s)\right) \\
& =0
\end{aligned}
$$

where $D_{i j}$ is the $(i, j)$-th element of $D$. By putting (A. 8) for $k=1,2, \ldots, n N$ together, we have (32).

\section{Appendix C: Robbins-Monro Algorithm [8]}

Consider the algorithm

$$
\zeta(l+1)=\zeta(l)-a(l) F(\zeta(l))+a(l) \kappa(l)
$$

where $\zeta(l) \in \mathbf{R}^{\gamma}$ is the state, $\kappa(l) \in \mathbf{R}^{\gamma}$ is the random variable, $a(l) \in \mathbf{R}_{+}$is the gain, and $F: \mathbf{R}^{\gamma} \rightarrow \mathbf{R}$ is a function. This algorithm is called the Robbins-Monro Algorithm.

For the algorithm, the following convergence result has been obtained (see, e.g., [8]).

Lemma 3: For the algorithm in (A.9), assume that there exists a vector $\zeta^{*} \in \mathbf{R}^{\gamma}$ such that $\zeta^{*}$ is an asymptotically stable equilibrium of the gradient system $\dot{\zeta}(\tau)=-F(\zeta(\tau))$. Then

$$
\lim _{l \rightarrow \infty} \zeta(l)=\zeta^{*} \quad \text { w.p. } 1,
$$

if the following conditions hold:

(C1) For a compact set $\mathbf{S} \subseteq \mathbf{R}^{\gamma}$ such that $\dot{\zeta}(\tau)=-F(\zeta(\tau))$ with $\zeta(0) \in \mathbf{S}$ results in $\zeta(\infty)=\zeta^{*}, \zeta(l) \in \mathbf{S}$ occurs infinitely often for almost all sample points of $k(l)(l=$ $0,1, \ldots)$.

(C2) $\lim _{l \rightarrow \infty} a(l)=0$ and $\sum_{l=0}^{\infty} a(l)=\infty$.

(C3) $\sup \|\zeta(l)\|<\infty$ w.p.1.

(C4) $\lim _{n \rightarrow \infty} \mathbb{P}\left(\sup _{m \geq n}\left\|\sum_{l=n}^{m} a(l) \kappa(l)\right\| \geq \eta\right)=0$ for any $\eta \in \mathbf{R}_{+}$.

\section{Appendix D: Proof that (C1)-(C4) hold}

It is clear that (B1) implies (C2) and that (B2) corresponds to $(\mathbf{C} 3)$. So we prove that $(\mathbf{C 1})$ and $(\mathbf{C 4})$ hold under (A1), (B1), and (B2).

\section{D.1 Proof for $(\mathbf{C 1})$}

From (B2), there exists an $M(x(0)) \in \mathbf{R}_{+}$satisfying

$$
\|x(t)\| \leq M(x(0)) \quad \forall t \in \mathbf{Z}_{0+} .
$$

Then, consider a compact set $\mathbf{S}$ such that

$$
\left\{x \in \mathbf{R}^{n N} \mid\|x\| \leq M(x(0))\right\} \subseteq \mathbf{S} .
$$

For this set, it follows that

(i) $x(t) \in \mathbf{S}$ occurs infinitely often for almost all sample points.

(ii) For the gradient system $\dot{\zeta}(\tau)=-\nabla J(\zeta(\tau)), \zeta(0) \in \mathbf{S}$ results in $\zeta(\infty)=x^{*}$

The former is given by (A-10) and (A-11). The latter is obtained by $\nabla J=2 P x+p$ and $P$ is positive-definite. Therefore, (C1) holds subject to (B2).

\section{D.2 Proof for $(\mathbf{C} 4)$}

First, let

$$
H(\sigma):=\sum_{s^{\prime}=s}^{\sigma} a\left(2 s^{\prime}+1\right) e\left(2 s^{\prime}+1\right)
$$


for $\sigma \geq s$. Since

$$
H(\sigma)=H(\sigma-1)+a(2 \sigma+1) e(2 \sigma+1),
$$

by definition, we have

$$
\begin{aligned}
& \mathbb{E}(H(\sigma) \mid H(\sigma-1)) \\
& =\mathbb{E}(H(\sigma-1)+a(2 \sigma+1) e(2 \sigma+1) \mid H(\sigma-1)) \\
& =H(\sigma-1)+a(2 \sigma+1) \mathbb{E}(e(2 \sigma+1) \mid H(\sigma-1)) .
\end{aligned}
$$

Then (36) means that

$$
\mathbb{E}(e(2 s+1) \mid x(2 s))=0 \forall(s, x(2 s)) \in \mathbf{Z}_{0+} \times \mathbf{R}^{n N}
$$

which, together with (37), gives

$$
\mathbb{E}(e(2 \sigma+1) \mid e(2 s+1))=0
$$

for $s=0,1, \ldots, \sigma-1$. So (A· 12), (A. 13), and (A-15) provide

$$
\mathbb{E}(H(\sigma) \mid H(\sigma-1))=H(\sigma-1),
$$

which implies that the stochastic process $H(\sigma)(\sigma=s, s+$ $1, \ldots)$ is a martingale. Then, in a similar way to [8], it follows from the Doob's martingale inequality that

$$
\begin{aligned}
\mathbb{P}\left(\sup _{\sigma \geq s}\left\|\sum_{s^{\prime}=s}^{\sigma} a\left(2 s^{\prime}+1\right) e\left(2 s^{\prime}+1\right)\right\| \geq \eta\right) \\
\leq \frac{1}{\eta^{2}} \mathbb{E}\left(\left\|\sum_{s^{\prime}=s}^{\infty} a\left(2 s^{\prime}+1\right) e\left(2 s^{\prime}+1\right)\right\|^{2}\right) .
\end{aligned}
$$

On the other hand, we can derive

$\frac{1}{\eta^{2}} \mathbb{E}\left(\left\|\sum_{s^{\prime}=s}^{\infty} a\left(2 s^{\prime}+1\right) e\left(2 s^{\prime}+1\right)\right\|^{2}\right) \leq \frac{4 n N \varepsilon}{\eta^{2} c^{2} d^{2}} \sum_{s^{\prime}=s}^{\infty} a^{2}\left(2 s^{\prime}+1\right)$

for the right-hand side of (A-16). In fact, since

$$
\begin{aligned}
& \mathbb{E}\left(e^{\top}\left(2 s_{1}+1\right) e\left(2 s_{2}+1\right)\right) \\
& =\mathbb{E}\left(\mathbb{E}\left(e^{\top}\left(2 s_{1}+1\right) e\left(2 s_{2}+1\right) \mid x(2), x(4), \ldots, x\left(2 s_{2}\right)\right)\right) \\
& =\mathbb{E}\left(e^{\top}\left(2 s_{1}+1\right) \mathbb{E}\left(e\left(2 s_{2}+1\right) \mid x(2), x(4), \ldots, x\left(2 s_{2}\right)\right)\right) \\
& =0
\end{aligned}
$$

for every $s_{1}, s_{2} \in \mathbf{Z}_{0+}\left(s_{1}<s_{2}\right)$ from (A- 14), we obtain

$$
\begin{aligned}
& \frac{1}{\eta^{2}} \mathbb{E}\left(\left\|\sum_{s^{\prime}=s}^{\infty} a\left(2 s^{\prime}+1\right) e\left(2 s^{\prime}+1\right)\right\|^{2}\right) \\
& =\frac{1}{\eta^{2}} \mathbb{E}\left(\sum_{s^{\prime}=s}^{\infty} a\left(2 s^{\prime}+1\right)\left\|e\left(2 s^{\prime}+1\right)\right\|^{2}\right) \\
& =\frac{1}{\eta^{2}} \sum_{s^{\prime}=s}^{\infty} a\left(2 s^{\prime}+1\right) \mathbb{E}\left(\left\|e\left(2 s^{\prime}+1\right)\right\|^{2}\right) .
\end{aligned}
$$

In addition, it follows from (A1), (6), (7), (13), (35), and (36) that

$$
\begin{aligned}
\mathbb{E}( & \left.\left\|e\left(2 s^{\prime}+1\right)\right\|^{2}\right) \\
= & \mathbb{E}\left(\left\|\phi\left(2 s^{\prime}+1\right)-\mathbb{E}\left(\phi\left(2 s^{\prime}+1\right) \mid x\left(2 s^{\prime}\right)\right)\right\|^{2}\right) \\
= & \mathbb{E}\left(\left\|\phi\left(2 s^{\prime}+1\right)\right\|^{2}\right) \\
& -\mathbb{E}\left(\left(2 \phi\left(2 s^{\prime}+1\right)-\mathbb{E}\left(\phi\left(2 s^{\prime}+1\right) \mid x\left(2 s^{\prime}\right)\right)\right)^{\top}\right. \\
= & \left.\mathbb{E}\left(\left\|\phi\left(2 s^{\prime}+1\right)\right\|^{2}\right)-\| \mathbb{E}\left(\phi\left(2 s^{\prime}+1\right) \mid x\left(2 s^{\prime}\right)\right)\right) \\
\leq & \mathbb{E}\left(\left\|\phi\left(2 s^{\prime}+1\right)\right\|^{2}\right) \\
= & \mathbb{E}\left(\frac{\left(J\left(q\left(x\left(2 s^{\prime}+1\right)\right)\right)-J\left(q\left(x\left(2 s^{\prime}\right)\right)\right)\right)^{2}}{c^{2} d^{2}}\left\|\alpha^{(-1)}\left(2 s^{\prime}\right)\right\|^{2}\right) \\
\leq & \frac{4 n N \varepsilon}{c^{2} d^{2}}
\end{aligned}
$$

for some $\varepsilon \in \mathbf{R}_{+}$such that $\mathbb{E}\left((J(q(x(t))))^{2}\right)<\varepsilon$ for every $t \in \mathbf{Z}_{0+}$ (note that (8) and (B2) guarantee the existence of $\varepsilon$ ).

Therefore, (A. 16) and (A. 17) provide

$$
\begin{aligned}
\mathbb{P}\left(\sup _{\sigma \geq s} \| \sum_{s^{\prime}=s}^{\sigma} a\left(2 s^{\prime}\right.\right. & \left.+1) e\left(2 s^{\prime}+1\right) \| \geq \eta\right) \\
& \leq \frac{4 n N \varepsilon}{\eta^{2} c^{2} d^{2}} \sum_{s^{\prime}=s}^{\infty} a^{2}\left(2 s^{\prime}+1\right) .
\end{aligned}
$$

Here, by considering that (B1) implies

$$
\lim _{s \rightarrow \infty} \sum_{s^{\prime}=s}^{\infty} a^{2}\left(2 s^{\prime}+1\right)=0
$$

it is proven that (C4) holds under (A1), (B1), and (B2).

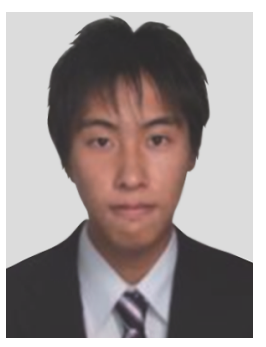

Yosuke Tanaka received his B.S. degree from Kyoto University, Japan, in 2012. He is currently a master course student in the Department of Systems Science, Kyoto University. 


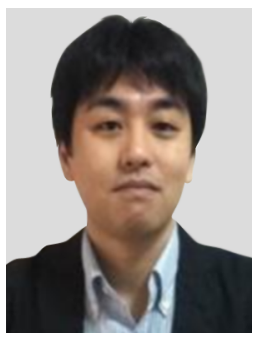

Shun-ichi Azuma received his B.E. degree in electrical engineering from Hiroshima University, Higashi Hiroshima, Japan, in 1999, and the M.E. and Ph.D. degrees in control engineering from Tokyo Institute of Technology, Tokyo, Japan, in 2001 and 2004, respectively. He was a Research Fellow with the Japan Society for the Promotion of Science Tokyo Institute of Technology, and a Visiting Scholar with the Georgia Institute of Technology, Atlanta, USA, from 2004 to 2005. He is currently an Associate Professor with the Department of Systems Science, Graduate School of Informatics, Kyoto University, Kyoto, Japan. His research interests include analysis and control of hybrid systems and biosystems.

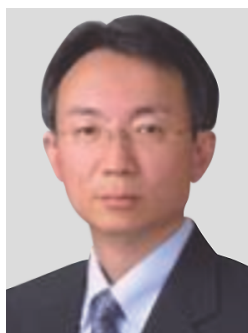

Toshiharu Sugie received his B.E., M.E., and $\mathrm{Ph} . \mathrm{D}$. degrees in engineering from Kyoto University, Japan, in 1976, 1978, and 1985, respectively. Since 1998, he has been a Professor at the Department of Systems Science, Kyoto University. He was a research member of Musashino Electric Communication Laboratory in Nippon Telegraph and Telephone Public Corporation, Japan (1978-1980), a Research Associate at the Department of Mechanical Engineering, University of Osaka Prefecture (1984-

1988), and an Associate Professor as the Department of Applied Systems Science, Kyoto University (1988-1997). His research interests are in robust control, identification for control, and their application to mechanical systems. He serves as an Editor of Automatica. He is an IEEE Fellow. 